\title{
Laser Transport System Vacuum Simulations and LED Atom Tracker
}

\author{
Jordan Aasman, CCI Intern
}

Mentors: Linda Valerio, Jesse Batko, and Beth Klein

August 7, 2020

This manuscript has been authored by Fermi Research Alliance, LLC under Contract No. DE-AC02-07CH11359 with the U.S. Department of Energy, Office of Science, Office of High Energy Physics. 


\begin{abstract}
MAGIS-100 is an experiment using atom interferometry on strontium atoms in a $100 \mathrm{~m}$ vacuum chamber. The laser travels through a laser transport system (LTS) before going down the chamber. Two projects were completed over the course of the internship. One project was conducting vacuum simulations on the LTS to ensure it meets the experimental pressure requirement of $10^{-11}$ torr. This was done by characterizing the pressure profile with variations in pump size, pump spacing, and orifice size. The initial design of the LTS included one $30 \mathrm{~L} / \mathrm{s}$ ion pump in the high vacuum (HV) region, a lens mount with holes of diameter 0.788 ", and three $150 \mathrm{~L} / \mathrm{s}$ ion pumps in the ultra-high vacuum (UHV) region. After adjusting these variables, it was concluded that the lens mount holes could be reduced to 0.200 " in diameter and one $150 \mathrm{~L} / \mathrm{s}$ ion pump in the UHV could be removed and still meet the experimental requirements. The other project was to design an LED atom tracker that shows the position of the strontium atoms in the $100 \mathrm{~m}$ shaft. This will help with public outreach by allowing visitors to have a visual of the experiment. An Arduino Uno and Arduino Due were programmed using classical physics formulas to direct the RGB LED strips to flash in sync with the falling atoms. The requirements for powering and connecting the hardware were calculated and recommendations for scaling the system to $100 \mathrm{~m}$ were made.
\end{abstract}

Introduction

$$
\text { Body }
$$

The Matter-wave Atomic Gradiometer Interferometric Sensor-100 (MAGIS-100) experiment uses atomic interferometry on strontium atoms as they fall in a vacuum chamber. The laser beam involved goes through a Laser Transport System (LTS) where it is amplified and made coherent before it descends the $100 \mathrm{~m}$ tall vacuum chamber. The LTS requires a vacuum on the order of 10E-11 torr so that the laser beam will remain stable enough for the experimental requirements. The scientists and engineers working on the project proposed a design for the LTS region with a certain number of ion pumps and an orifice. One project was to conduct vacuum simulations on this design to verify it meets the experimental requirements and optimize the orifice size and pumping speed. The project's objective was met by obtaining vacuum data on the proposed LTS design and recommending the use of one $30 \mathrm{~L} / \mathrm{s}$ ion pump in the high vacuum (HV) region and two $150 \mathrm{~L} / \mathrm{s}$ ion pumps in the ultra-high vacuum region (UHV) while reducing the size of the holes in the orifice.

As the strontium atoms travel up and down the vacuum chamber, it was proposed that there should be a way to display their progress. This will help with public outreach by allowing spectators to see where the strontium atoms are when the experiment runs. The LED Atom Tracker project was to design a system that will accomplish this task. A series of LED strips were programmed to flash in sync with the strontium atoms' trajectory. There are three science modes that MAGIS-100 will run in. One is where the strontium atoms are launched from the bottom of the $100 \mathrm{~m}$ shaft. This will be the hardest mode to program in the circuit. The second science mode is where the strontium atoms are launched from the bottom of the shaft and about midway up the shaft simultaneously and only travel about $40 \mathrm{~m}$. This will be the easiest science mode to configure. The last science mode is where the strontium atoms are dropped from the top of the $100 \mathrm{~m}$ shaft. This will be in between the other science modes in terms of difficulty. 


\section{Progress}

The basics of vacuum, vacuum pumps, and the software used for conducting vacuum simulations were studied to gain an understanding of vacuum systems [1][2]. The primary characteristics that influence a vacuum system are conductance, effective pumping speed, outgassing rate, and pressure. After learning the fundamentals, the vacuum simulations began.

The steps taken for analyzing the vacuum system were as follows. First a vacuum tube was modeled in NX, saved as an STP file, and exported in .txt format to the local computer. The file was saved as a .STL file and imported into Molflow, a test-particle Monte-Carlo simulator for ultra-high-vacuum systems. A sample simulation in Molflow is shown in Figure 1. This simulation was used to find the conductance of the tube. The result was cross checked with a preformatted Excel spreadsheet provided by Jesse Batko, a Fermilab engineer, and hand calculations using formulas for vacuum systems [3].

The ANSYS software doing the vacuum simulation actually does a thermal simulation. The Excel spreadsheet "APDL Vacuum Sim," also created by Jesse Batko, converts the vacuum values into thermal values. The effective pumping speed of the ion pumps was used to find the conductance of the ion pumps. The outgassing rate for stainless steel was $6.7 \mathrm{E}-13$ torr. The conductance values of the tube and ion pumps, the perimeter of each part, and the outgassing rate were input into the "APDL Vacuum Sim" spreadsheet. In this spreadsheet, a simulation was setup by inputting nodes on an XY coordinate plane and assigning each node a conductance value according to the part it represented. An example of the spreadsheet is shown in Figure 2. For example, the first simulation that ran consisted of 10 nodes and is shown in Figure 3 . The result of the ANSYS simulation resulted in a value for each node in kelvins. These values were input into another Excel template called "Comparing Runs," also created by Jesse Batko, that converted the temperature (kelvins) values into pressure (torr). The conversion from kelvins to pascals is one to one, so the conversion is simple. The "Comparing Runs" spreadsheet is shown in Figure 4. A graph of the pressure over distance from the first to the final node was generated and an example is shown in Figure 5. This procedure was repeated with different variables and the most optimized system was found.

In the initial design there were four ion pumps and sixteen 0.788 " holes in the orifice. The variables in the vacuum system were ion pump speed, placement, and diameter of holes in the orifice. These variables were adjusted and optimized. The current design has 3 ion pumps and sixteen 0.200 " holes in the orifice. By removing one ion pump, the cost was decreased by approximately $\$ 10,000$, and the experimental requirement of $10 \mathrm{E}-11$ torr was still met.

For the of the LED Atom Tracker project, circuits were programmed and tested using the Arduino Integrated Development Environment (IDE) paired with an Arduino Uno that was connected to a breadboard, jumper wires, and RGB LED strips. WS2815 LED strips were chosen because they are $12 \mathrm{~V}$, able to create up to 16 million colors, easily connected into a longer strip, and compatible with Arduino microcontrollers.

The steps taken in the LED atom tracker project were as follows. First, classical physics functions that modeled the strontium atoms' trajectory were calculated using a Mathematica Notebook created by Sam Carman, a Stanford graduate student. Next, these functions were programmed into TinkerCAD, an online electronics simulation application. An example of the TinkerCAD environment is shown in Figure 6. Then, the code from TinkerCAD was copied to the Arduino IDE as shown in Figure 7. Next, the circuit was implemented on a real Arduino Uno connected to a 5m strip of WS2815 RGB LED lights. An example of the circuit connection to the Arduino Uno is shown in Figure 8. An alternative to the Arduino Uno was the Arduino Due. A 
connection with an Arduino Due is shown in Figure 9. The circuit was run and a picture of what the end result looked like is shown in Figure 10.

The Adafruit_NeoPixel.h library functions were used to program the LED strip [4]. This library was chosen over the FastLED library because the FastLED library was not available in TinkerCAD and the Adafruit NeoPixel library was easier to start with; as more progress is made, it might be determined that the FastLED library has more features relevant to the project [5].

The power requirements for the LED strips was calculated. From the reference documentation, each color in an LED uses $20 \mathrm{~mA}$ of current when turned on [6]. Each LED has red, green, and blue which makes each LED use a max total of $60 \mathrm{~mA}$ when all colors are turned on max brightness. For caution, this is the power consumption assumed in the calculations. There are $60 \mathrm{~mA}$ of current per LED times 60 LEDs per meter times 100 meters divided by $1000 \mathrm{~mA}$ to get a maximum of 360 A of current for the whole $100 \mathrm{~m}$ system of LEDs, if all LEDs are turned on at maximum brightness at once. The minimum current requirement is one third of $360 \mathrm{~A}$ which is $120 \mathrm{~A}$ if only one of red, green, or blue is turned on in every LED at once. The WS2815 strip runs at $12 \mathrm{~V}$. Multiplying $12 \mathrm{~V}$ by $120 \mathrm{~A}$ returns $1440 \mathrm{~W}$ for the minimum power consumption and $12 \mathrm{~V}$ times $360 \mathrm{~A}$ to get $4320 \mathrm{~W}$ for the maximum power consumption. It is likely that because only a few LEDs will be turned on at one time, that the power consumption will be less. Using a multimeter, the voltage drop was measured when the $5 \mathrm{~m}$ strip was set to white (red, green, and blue turned on in each pixel) at full brightness. The voltage dropped from $11.90 \mathrm{~V}$ where the strip connected to power down to $9.80 \mathrm{~V}$ at the end of the $5 \mathrm{~m}$ strip. There was no visual effect on the pixels towards the end of the strip. When the program with only one LED lit up at a time, the voltage at the start of the strip was $12.50 \mathrm{~V}$ and at the end of the strip it was $12.10 \mathrm{~V}$.

It is generally recommended to power inject every $10 \mathrm{~m}$ when using $12 \mathrm{~V}$ LED strips [7]. Because the voltage drop of the current circuit is small, power injection might only be needed in longer intervals of LED strips. It is recommended to see how the $12 \mathrm{~V}$ LED strip handles the Stanford 10m experiment and use those observations to inform the decisions regarding the power connections on MAGIS-100.

Two ways to do power injection were recommended. The first way was to use a barrel jack connector attached to a $12 \mathrm{~V}$ power supply transformer as shown in Figures 8 and 9. The red and white wires from the LED strip were connected to the positive and negative terminals of the barrel jack connector respectively. A 1000 micro-Farad capacitor was connected as well with the longer leg of the capacitor connecting to the positive terminal and the shorter leg connecting to the negative terminal. The other way was through wiring the LED strips' power wires directly to a $12 \mathrm{~V}$ power supply transformer as shown in Figure 11. From the wire that is plugged into the wall, the black wire connected to " $\mathrm{L}$ " for live, the white wire connected to " $\mathrm{N}$ " for neutral, and the green wire connected to ground. This powered the power supply with the voltage used, in this case $12 \mathrm{~V}$. The two power lines from the LED strip were connected, too. The red wire was connected to " $+\mathrm{V}$ " for positive voltage and the white wire was connected to "-V" for ground.

The microcontroller memory requirements were calculated. According to an Arduino tutorial, each pixel requires 3 bytes of RAM [8]. The Arduino Uno has an AVR-based microcontroller with 32 kilobytes of flash memory, 2 kilobytes of SRAM, and 1 kilobyte of electrically erasable programmable read only memory (EEPROM). Flash memory is where the Arduino sketch is stored, SRAM is where the sketch creates and manipulates variables when it runs, and EEPROM is memory that can be used to store long term information [9]. The Arduino Due has an ARM based microcontroller which allows it to have more memory and higher CPU 
speed. It has 512 kilobytes of flash memory, 96 kilobytes of SRAM, and no EEPROM. When the current program for science mode 3 was compiled for the Arduino Uno, the compiler said that only 2668 bytes out of 32 kilobytes (about $8 \%$ ) of the flash memory was used and only 41 bytes of SRAM out of 2 kilobytes (about 2\%) was used, no matter whether there were 300 LEDs as in the $5 \mathrm{~m}$ strip or 6000 LEDs as in the $100 \mathrm{~m}$ strip. It is recommended to use the Arduino Uno first with the final program to see if it will be capable of handling all 6000 LEDs. If it cannot, it is recommended to upgrade to the Arduino Due to handle all of the memory requirements. There will be no difference in connecting either board to the LED strips as shown in Figures 8 and 9. It seems that a single Arduino microcontroller will be sufficient for this project, so it is not recommended to implement multiple microcontrollers at once, such as having two or three Arduinos down the shaft communicating with the master Arduino. This will add unnecessary complications to the project.

The LED strips will need to be mounted on the vacuum shaft to show where the strontium atoms are. It is recommended to use the adhesive backing on the LED strips to stick to the shaft and use zip ties to hold the strips in place as a backup. The effect of the strips being connected to the shaft were not analyzed.

The maximum safety rating for the WS2815 LED strips is IP67. This means that the strip is waterproof up to $1 \mathrm{~m}$ for 30 minutes and dustproof from all debris [10]. There is not a one to one correlation with a NEMA rating. An exact safety rating required for the LED strips to be installed in the MINOS shaft was not given.

\section{Future Work}

A new objective during the vacuum simulation project was to make a more in-depth simulation on irregular geometries such as a mirror box. This could be done by using an ANSYS workbench steady state thermal simulation with various parameters such as heat flow, convection, and other thermal simulation properties. This kind of simulation will show a 3D model of the mirror box that displays a clearer illustration of what its pressure profile is. The future work to be done with this idea is to find a correlation between the current ANSYS simulations in the "APDL Vacuum Sim" spreadsheet and the steady state thermal simulations to get a baseline for the conversion between vacuum and thermal units.

It is possible that there are direct vacuum simulation software programs available, but they were not included in this project. In the future, there could be a way to do direct vacuum simulations. Software that does direct vacuum simulations could also have features to do the indepth 3D model of the vacuum system and be used instead of the idea presented above.

The end goal for the LED Atom Tracker is to have the person running the experiment select the science mode and press a button that starts the LEDs and experiment simultaneously. The rough draft circuit for this to happen on is shown in Figure 12.

An idea to use a sturdier way for connecting the wires from the LED strips to the Arduino might be considered. One idea would be to solder the WS2815 wires to the pins on the Arduino. Going forward, the software programs for all three science modes will be revised and have features added to them. It was demonstrated that it is possible to program two sections of the LED strip to run simultaneously, so the second science mode will be able to run properly. The current method of starting the program is on a button press. This prompts the LED strip to flash in one science mode at a time. It might be considered that multiple buttons connect to the Arduino on separate data pins that correspond to each science mode. 
Impact on Laboratory

These projects contributed to the MAGIS-100 collaboration. MAGIS-100 is part of the Fermi Quantum Initiative which seeks to advance quantum science in the United States. The work done would have been completed by another engineer if the intern had not done the projects.

Thank you to the Gordon and Betty Moore Foundation and the DOE Office of Science's Office of High Energy Physics QuantISED program for funding the primary research project.

\section{Conclusions}

For the vacuum simulations project, it was found that tubes with a wider diameter and a shorter length have a higher conductance. This is observed from the equation for conductance and is intuitive if thought about with reference to a hose. It is easier for water to travel through a wider, shorter hose than a longer, narrower one. The goal of the vacuum system is to reduce the number of impurities, ergo increasing the vacuum in the LTS. In order to create a higher vacuum, the smaller the conductance of the vacuum system, the better. An orifice was added to the mirror box to reduce the conductance even more. An orifice that has holes with smaller diameters have a lower conductance which helps lower the pressure in the vacuum system. It was found that a combination of three ion pumps and an orifice of 160.2 " holes in the mirror box was the most cost-effective solution that still met experimental requirements.

For the LED atom tracker project, the flashing LED strips on the shaft will add a nice aesthetic touch to the complex experiment when it runs. The LED strips connect to any Arduino and are easily programmable. The baseline for what the connections might look like and how the programs will run have been outlined in this document. The only work left is to scale up to $100 \mathrm{~m}$ and place the LEDs on the vacuum shaft.

References

1. Anderson, K., 2015. Vacuum With ANSYS.

2. Anderson, T., 2006. Accelerator Vacuum 101- Made Easy??

3. Lafferty, J. M. Foundations of Vacuum Science and Technology. New York: Wiley, 1998. Print.

4. https://github.com/adafruit/Adafruit NeoPixel

5. https://github.com/FastLED/FastLED

6. http://spikerlights.com/pwrinjection.aspx

7. https://www.flexfireleds.com/led-strip-light-voltage-drop-what-is-voltage-drop/

8. https://learn.adafruit.com/adafruit-neopixel-uberguide/arduino-library-use

9. https://www.arduino.cc/en/Tutorial/Memory

10. http://www.dsmt.com/resources/ip-rating-chart/ 
Participants

Appendix

\begin{tabular}{|l|l|l|l|}
\hline Name & Institution & Role & Description \\
\hline Jordan Aasman & $\begin{array}{l}\text { Fayetteville Technical } \\
\text { Community College }\end{array}$ & Undergraduate Intern & $\begin{array}{l}\text { Conducted vacuum } \\
\text { simulations on the LTS } \\
\text { and designed an LED } \\
\text { atom tracker }\end{array}$ \\
\hline Kelsey Scheidt & College of DuPage & Undergraduate Intern & $\begin{array}{l}\text { Designed an atom } \\
\text { source mount to support } \\
\text { the atom sources }\end{array}$ \\
\hline Linda Valerio & Fermilab & $\begin{array}{l}\text { Project Engineer/Senior } \\
\text { Mechanical Engineer }\end{array}$ & $\begin{array}{l}\text { Worked with scientists } \\
\text { on experimental } \\
\text { requirements and } \\
\text { designs }\end{array}$ \\
\hline Jesse Batko & Fermilab & $\begin{array}{l}\text { Staff Mechanical } \\
\text { Engineer }\end{array}$ & $\begin{array}{l}\text { Worked with scientists } \\
\text { on experimental } \\
\text { requirements and } \\
\text { designs }\end{array}$ \\
\hline Beth Klein & Fermilab & Engineer & $\begin{array}{l}\text { Supported instruction on } \\
\text { how to conduct vacuum } \\
\text { simulations and design } \\
\text { parts in NX }\end{array}$ \\
\hline
\end{tabular}

Scientific facilities

There were no scientific user facilities utilized as part of the project activities.

Notable outcomes

The notable outcome of the projects was the poster that was presented at the CCI/SULI Intern Poster Session. 


\section{Figures}

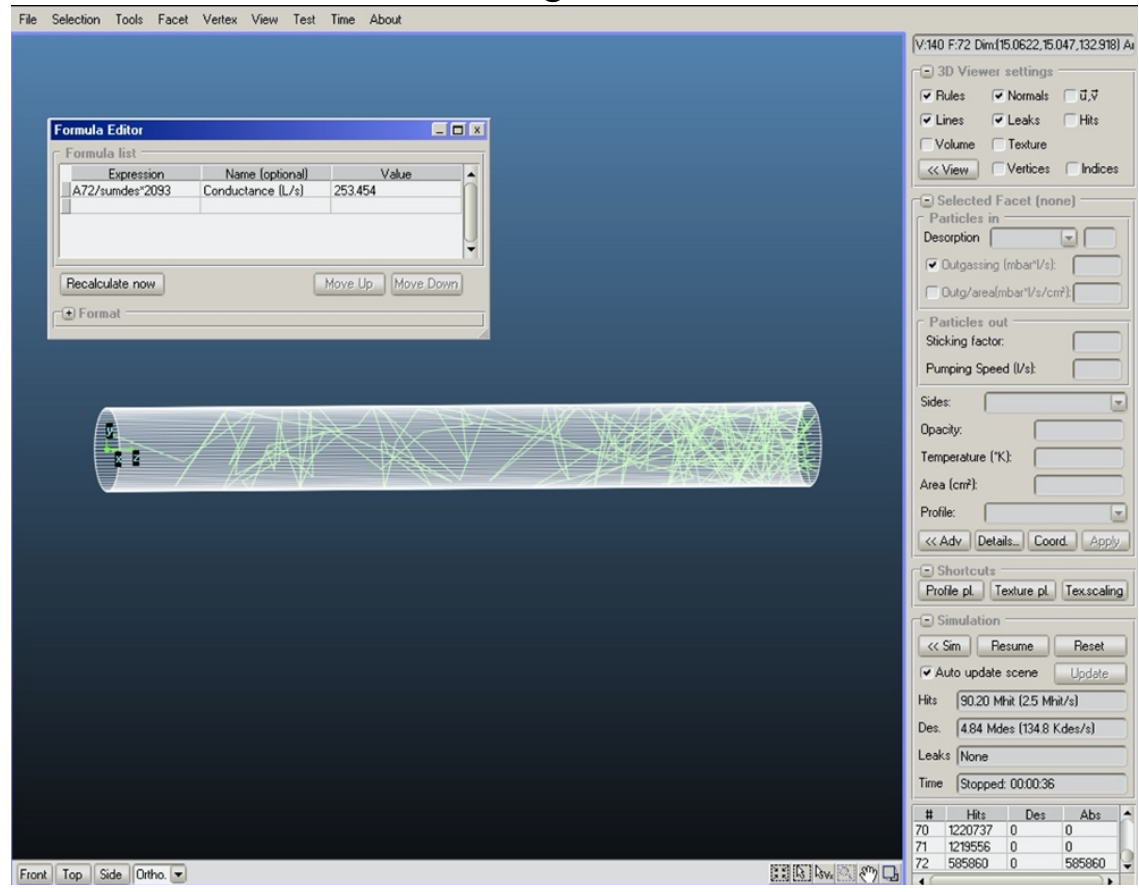

Figure 1

This figure shows a vacuum simulation on a stainless-steel tube in Molflow. The tube geometry was imported from NX as an STL file. The simulation is setup by designating a facet to be the one where particles go in and another facet to be the one where particles go out. In the toolbar, there is a calculator feature where the conductance equation is input. As the simulation runs, the conductance is seen as the result of the equation.

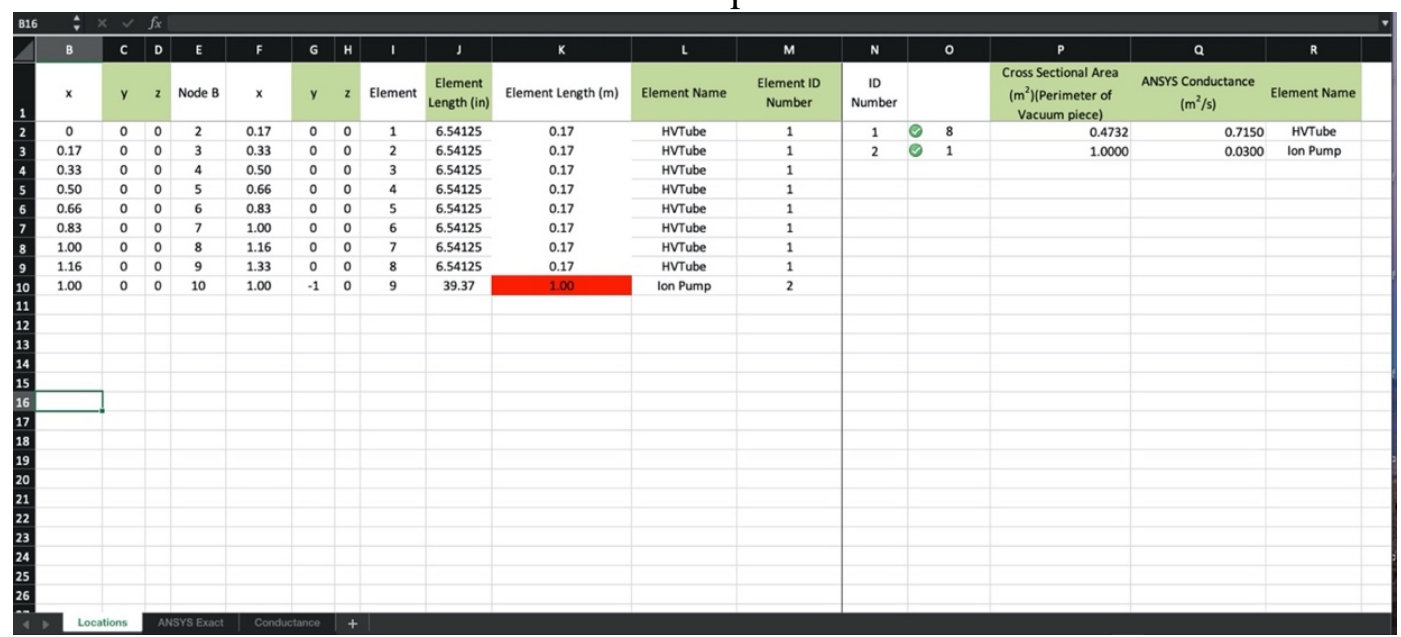

Figure 2

This figure shows the spreadsheet used to input the LTS geometry and characteristics such as conductance, pumping speed, perimeter, and outgassing rate. The spreadsheet then generates the code to be uploaded into ANSYS. 


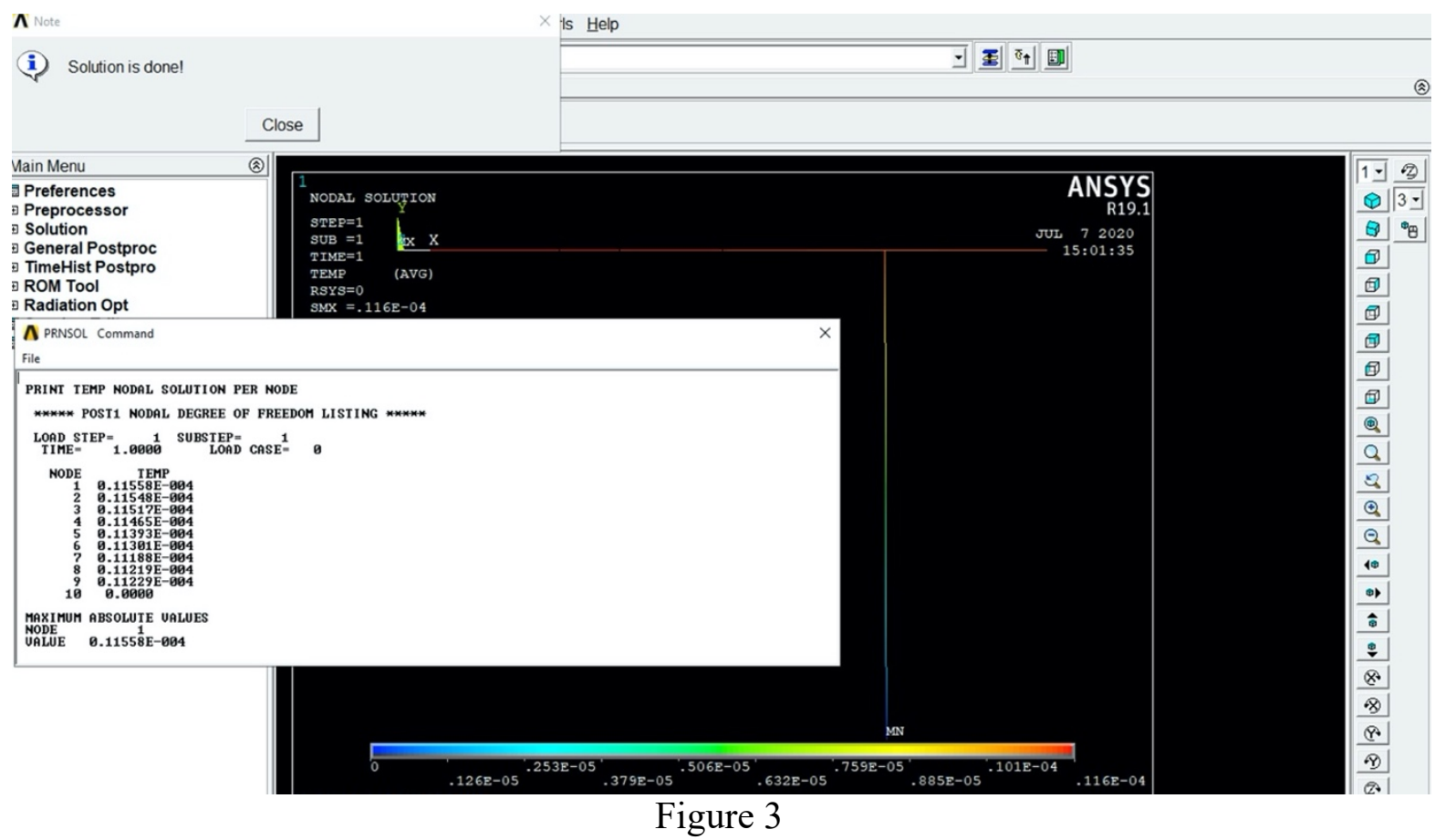

This figure shows an ANSYS thermal simulation. There is an illustration of the LTS and a table with the list of nodes and the temperature at each node.

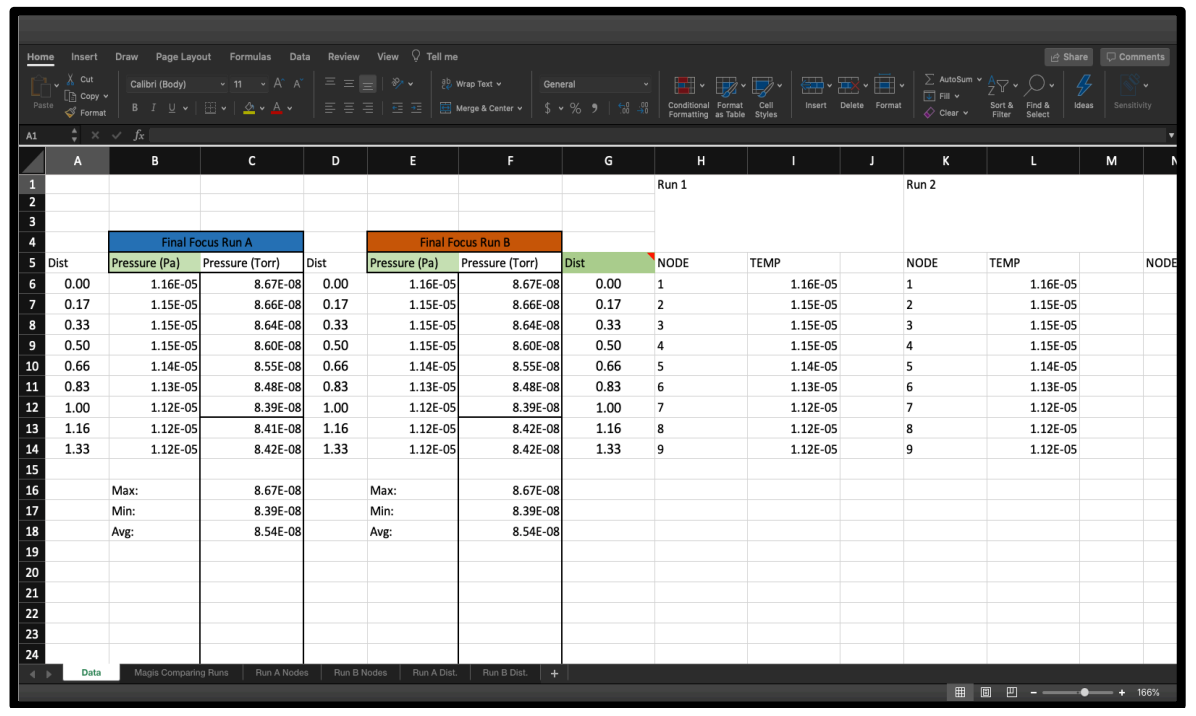

Figure 4

This figure shows the "Comparing Runs" spreadsheet. The results from the ANSYS simulation were input into it and converted back to vacuum units. 


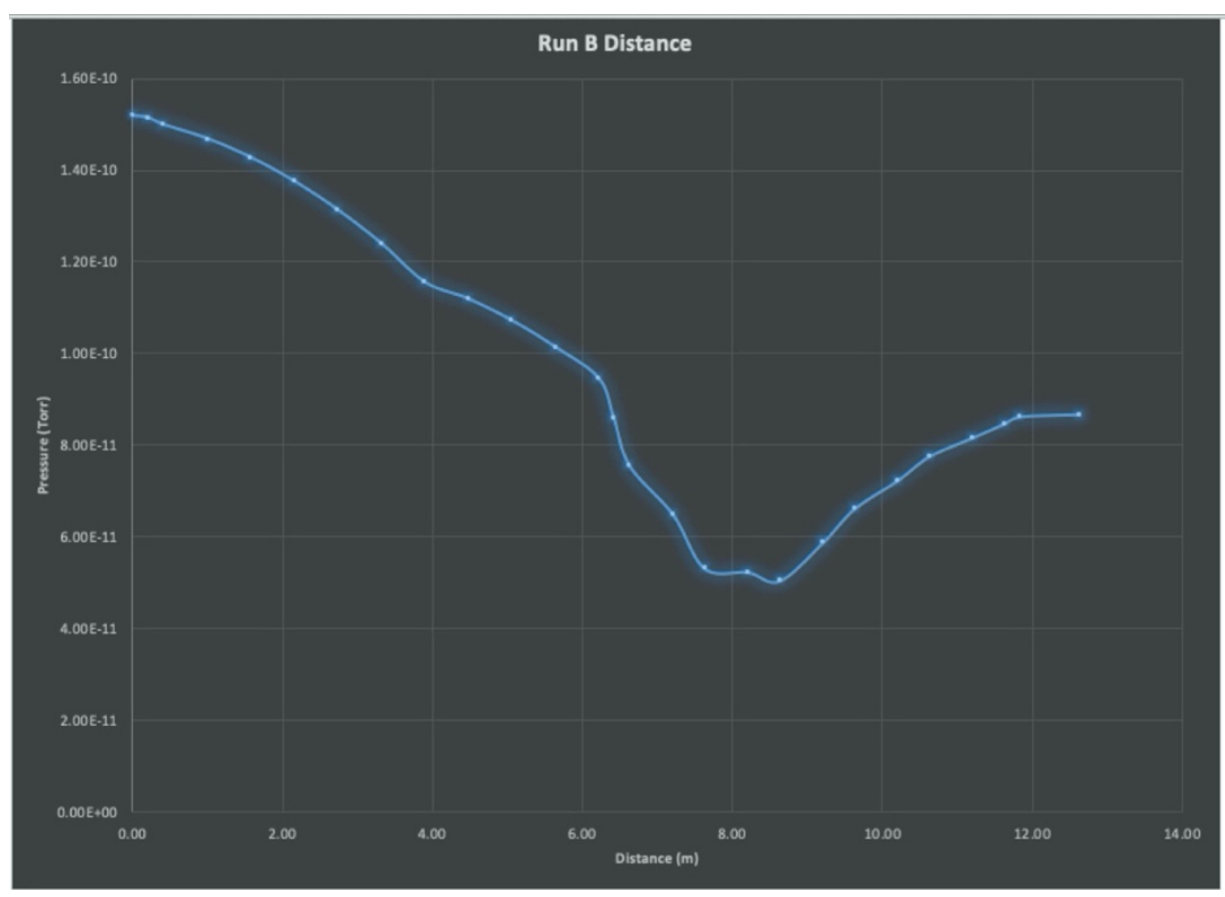

Figure 5

This figure shows the graph that was generated by the "Comparing Runs" spreadsheet. It shows the pressure at each node from the laser hutch to the top of the vacuum shaft. The pressure decreases as the distance from the laser hutch increases and passes through the orifice and ion pumps.

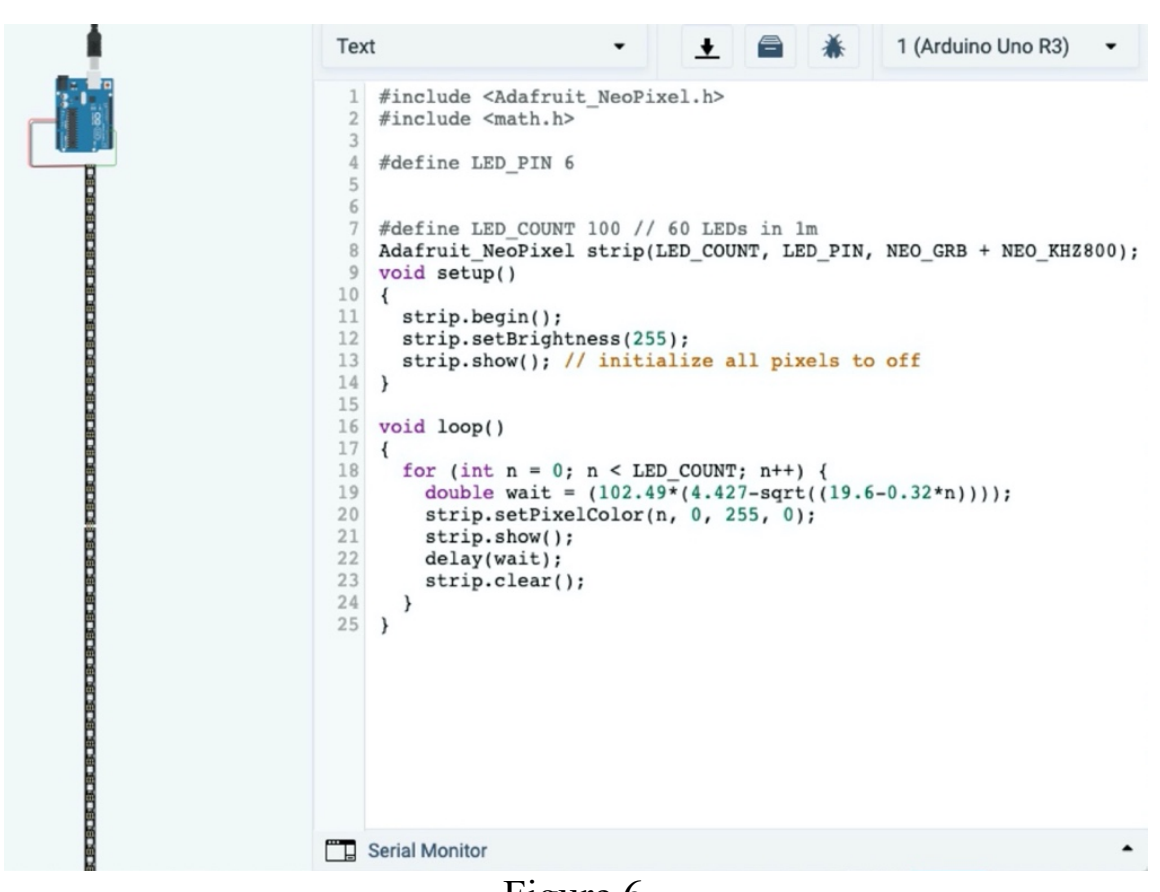

Figure 6

This figure shows the TinkerCAD development environment. The circuit is created by clicking and dragging parts together. The code editor allows for the use of the Adafruit NeoPixel library so the design for the LED Atom Tracker could be simulated. 


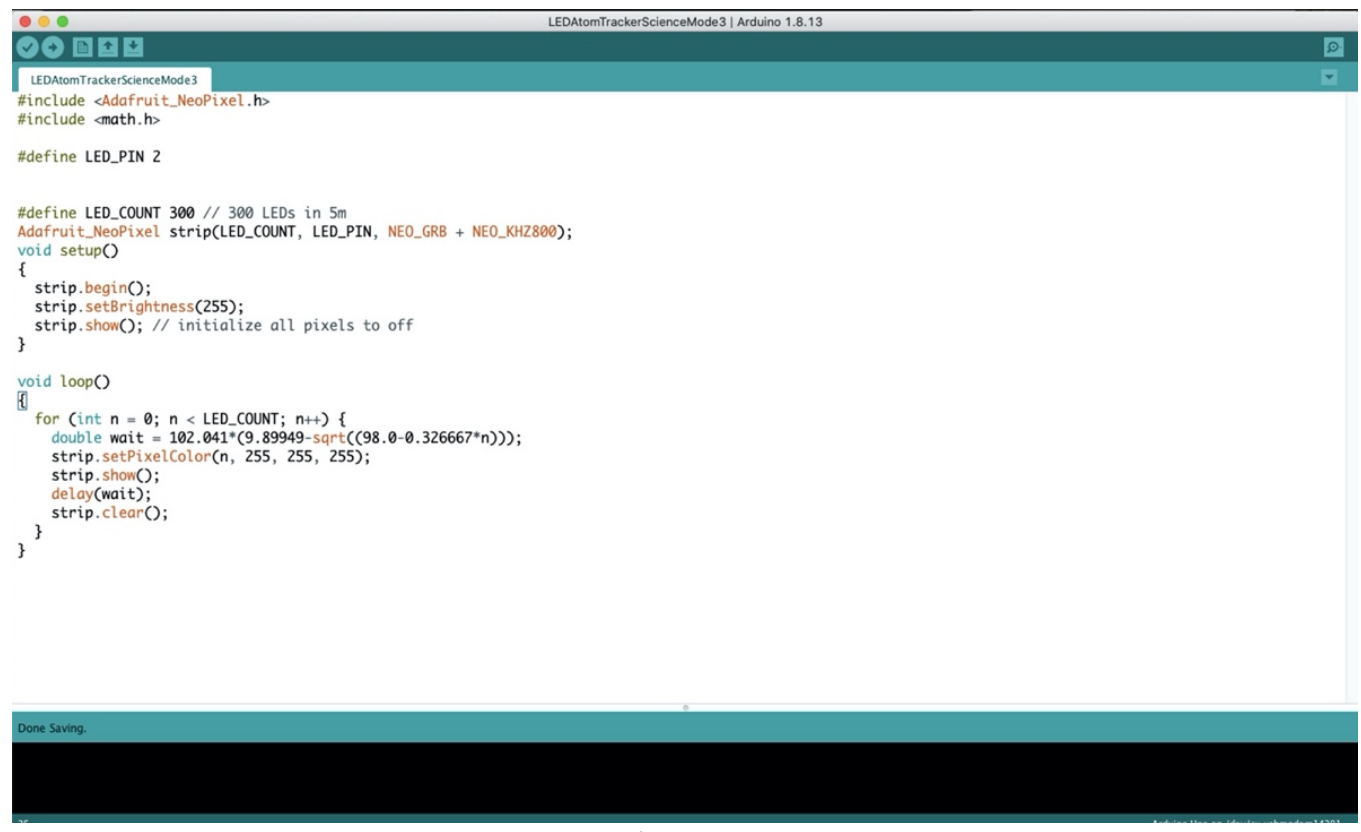

Figure 7

This figure shows the Arduino IDE with the program for science mode 3. The "wait" variable was calculated using the function from the Mathematica notebook. The code was compiled and uploaded to the Arduino Uno board.

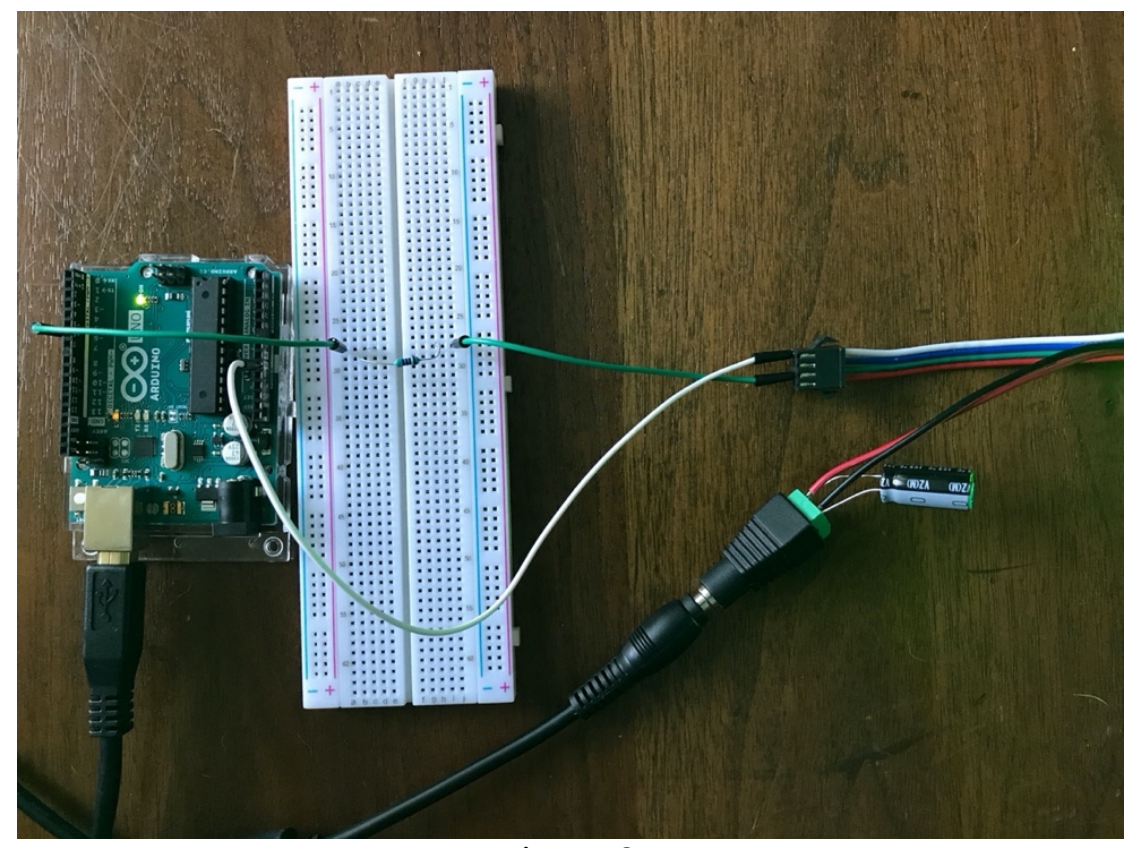

Figure 8

This figure shows the physical connection of the LED strip to the Arduino Uno. There is a $470 \mathrm{Ohm}$ resistor between the data pin on the Arduino and the data pin on the LED strip. The Arduino pin used is data pin 6. The barrel jack is connected to the power wires of the LED strip and the power source. A 1000 micro farad capacitor is connected across the terminals in order to prevent a power surge. 


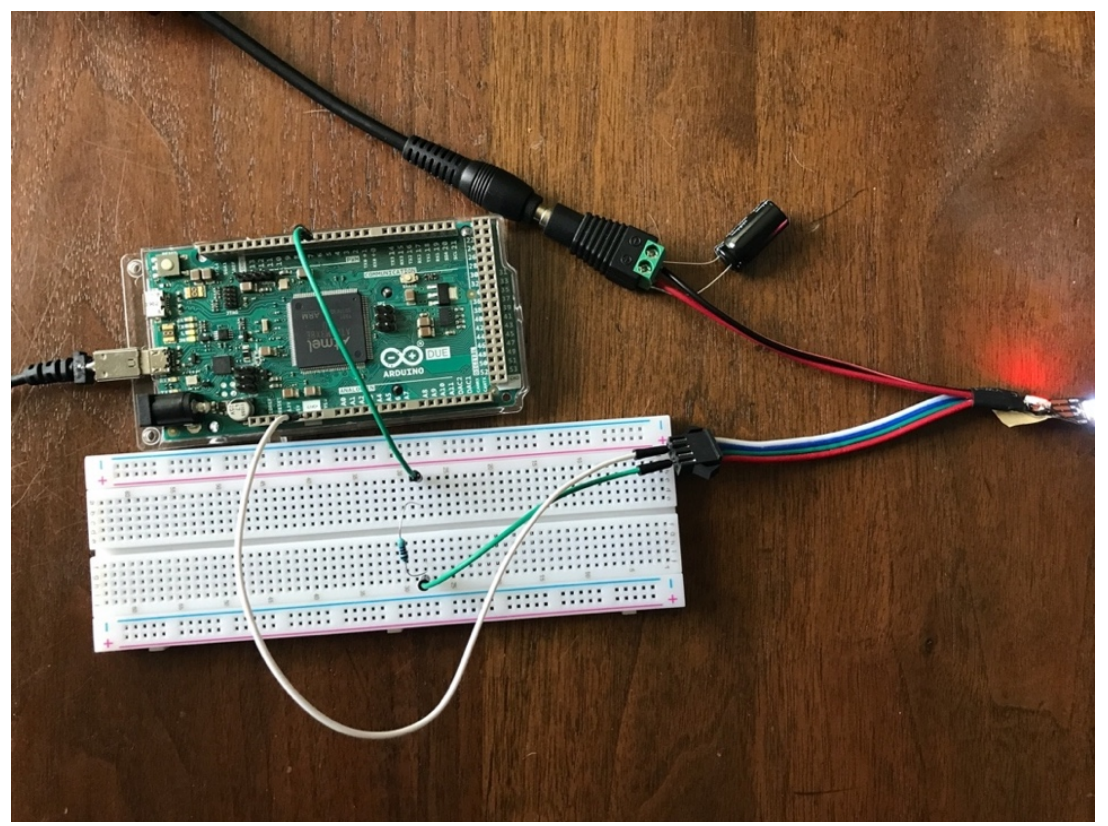

Figure 9

This figure shows the physical connection to the Arduino Due. There is a $470 \mathrm{Ohm}$ resistor between the data pin on the Arduino and the data pin on the LED strip. The Arduino pin used is data pin 6. The barrel jack is connected to the power wires of the LED strip and the power source. A 1000 micro farad capacitor is connected across the terminals in order to prevent a power surge. 


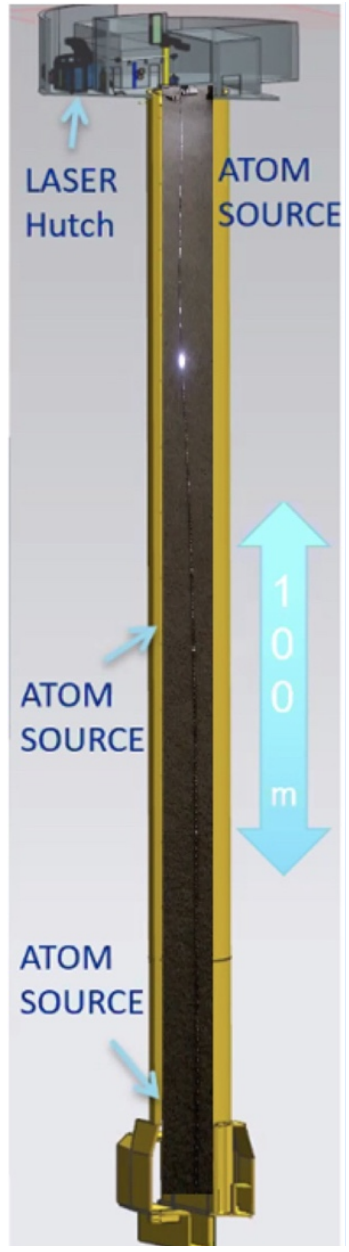

Figure 10

This figure shows what science mode 3 will look like when the LED strip is mounted to the vacuum shaft. The light will flash down the strip showing where the strontium atoms are during the experiment. 


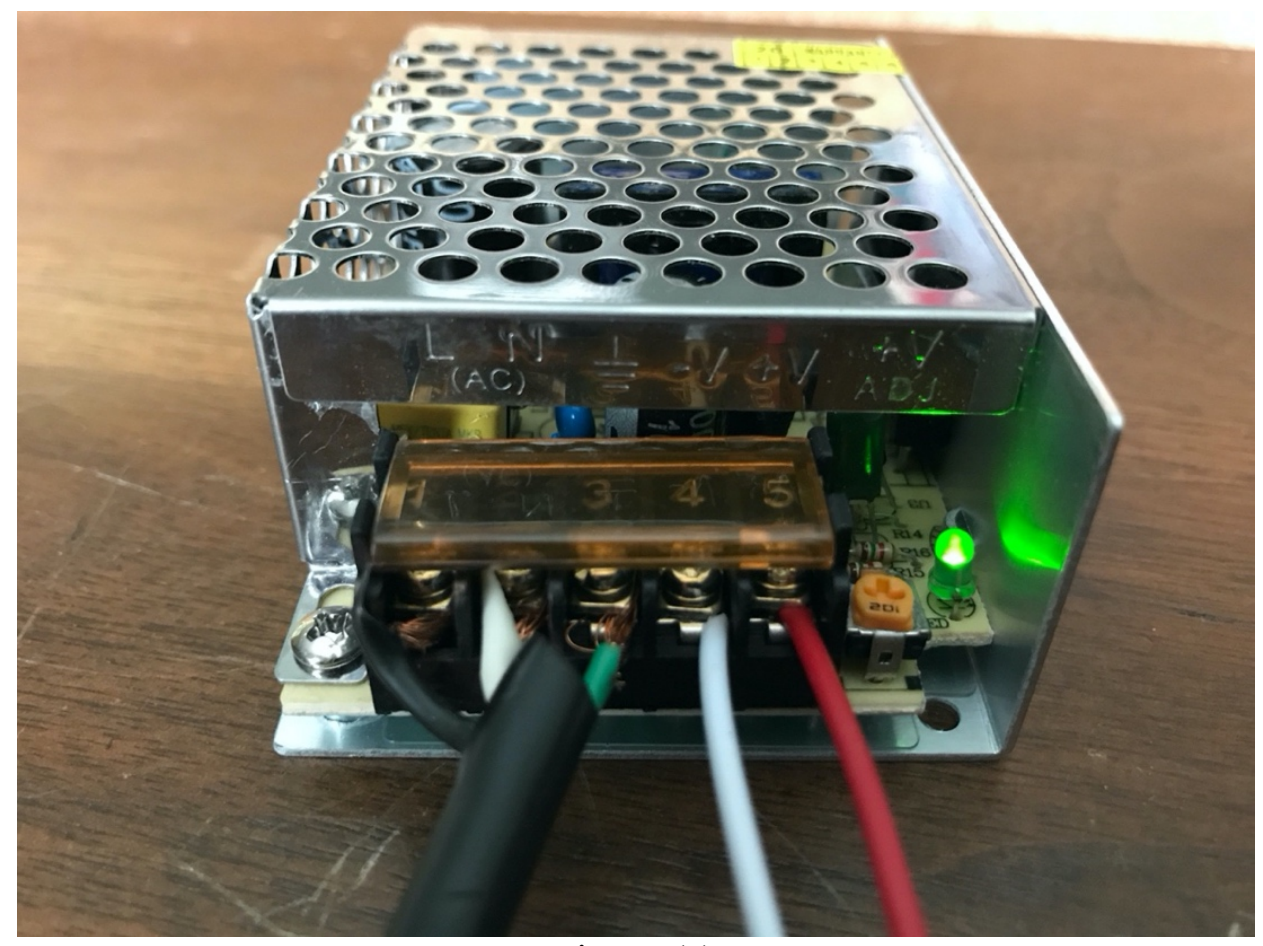

Figure 11

This figure shows the connection to the end of the LED strip where power will be injected. Power injection will be necessary along the 100m chain of LED lights so that each LED will have power. The wire supplying power is connected on the left three terminals. The black wire is connected to "L," the white wire is connected to "N," and the green wire is connected to ground.

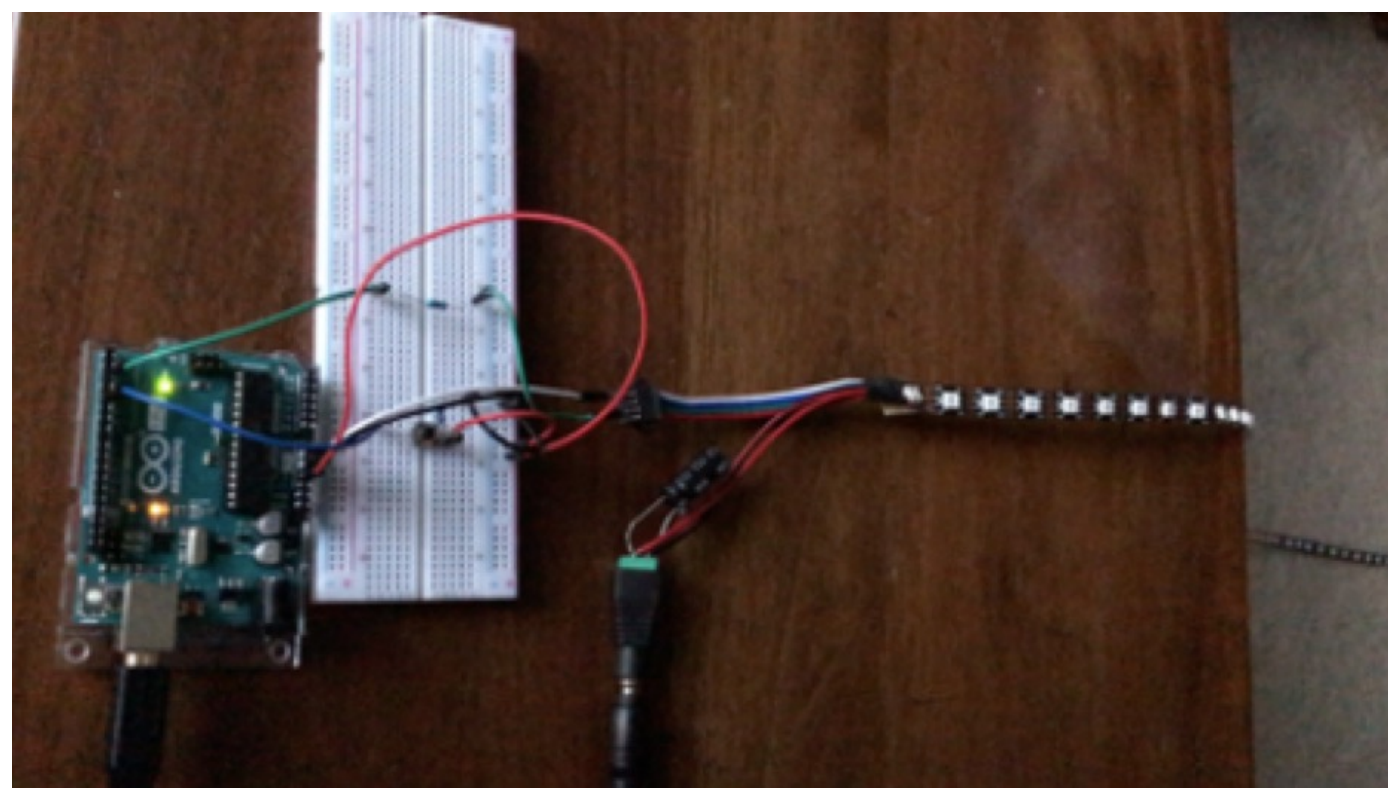

Figure 12

This figure shows a circuit with a button in it. The circuit is programmed to start when the button is pressed. The end goal of the LED Atom Tracker project is to have a button to start each of the different science modes going in the experiment and LED strips at the same time. 